\title{
Lnk constrains myeloproliferative diseases in mice
}

\author{
Alexey Bersenev, ${ }^{1}$ Chao Wu, ${ }^{1}$ Joanna Balcerek, ${ }^{1}$ Jiang Jing, ${ }^{1}$ Mondira Kundu, ${ }^{2}$ \\ Gerd A. Blobel,1,3 Kudakwashe R. Chikwava, ${ }^{4}$ and Wei Tong ${ }^{1,3}$

\begin{abstract}
1Division of Hematology, Children's Hospital of Philadelphia, Philadelphia, Pennsylvania, USA. 2Department of Pathology, St. Jude Children's Research Hospital, Memphis, Tennessee, USA. ${ }^{3}$ Department of Pediatrics, University of Pennsylvania School of Medicine, Philadelphia, Pennsylvania, USA. “Department of Pathology, Children’s Hospital of Philadelphia, Philadelphia, Pennsylvania, USA.
\end{abstract}

\begin{abstract}
Hematopoietic stem and progenitor cell (HSPC) expansion is regulated by intrinsic signaling pathways activated by cytokines. The intracellular kinase JAK2 plays an essential role in cytokine signaling, and activating mutations in JAK2 are found in a number of hematologic malignancies. We previously demonstrated that lymphocyte adaptor protein (Lnk, also known as Sh2b3) binds JAK2 and attenuates its activity, thereby limiting HSPC expansion. Here we show that loss of Lnk accelerates and exacerbates oncogenic JAK2-induced myeloproliferative diseases (MPDs) in mice. Specifically, Lnk deficiency enhanced cytokine-independent JAK/STAT signaling and augmented the ability of oncogenic JAK2 to expand myeloid progenitors in vitro and in vivo. An activated form of JAK2, unable to bind Lnk, caused greater myeloid expansion than activated JAK2 alone and accelerated myelofibrosis, indicating that Lnk directly inhibits oncogenic JAK2 in constraining MPD development. In addition, Lnk deficiency cooperated with the $B C R / A B L$ oncogene, the product of which does not directly interact with or depend on JAK2 or Lnk, in chronic myeloid leukemia (CML) development, suggesting that Lnk also acts through endogenous pathways to constrain HSPCs. Consistent with this idea, aged $\mathrm{Lnk}^{-/}$mice spontaneously developed a CML-like MPD. Taken together, our data establish Lnk as a bona fide suppressor of MPD in mice and raise the possibility that Lnk dysfunction contributes to the development of hematologic malignancies in humans.
\end{abstract}

\section{Introduction}

JAK2 plays an essential role in the signaling of receptors for many cytokines, which include thrombopoietin (Tpo), erythropoietin (Epo), and granulocyte-CSF (G-CSF) (1). JAK2-deficient mice die of anemia at embryonic day 12.5, and their fetal liver-derived hematopoietic cells fail to respond to Tpo, Epo, or G-CSF. Ligandbound cytokine receptors activate JAK2, which in turn phosphorylates the cytoplasmic tail of the receptors and triggers a cascade of signaling events (2). These signaling events involve a variety of positive mediators, such as Stats, PI3K/Akt, and MAPK. The receptor/JAK2 complexes also activate multiple negative regulators that provide checks and balances at multiple steps of cytokine receptor signal transduction to ensure a tightly controlled cellular response and prevent oncogenic transformation (3). One of these cytokine signaling attenuators is the lymphocyte linker (Lnk) protein (4).

Lnk is a member of an adaptor protein family that possesses a number of protein-protein interaction domains: a proline-rich amino-terminus, a pleckstrin homology $(\mathrm{PH})$ domain, a Src homology 2 (SH2) domain, and many potential tyrosine phosphorylation motifs (4). Lnk-deficient mice show profound perturbations in hematopoiesis, including a 3 -fold increase in circulating wbc and platelets $(5,6)$, accumulation of pro/pre and immature B cells in the BM and spleen, and expansion of the HSC pool with enhanced self-renewal (7-9). Along with others, we have previously identified Lnk as a negative regulator for Tpo receptor-mediated (TopR is also referred to as myeloproliferative leukemia virus proto-oncogene $[\mathrm{Mpl}]$ ) signaling pathways in both megakaryopoiesis and HSCs (8-11). Lnk constrains HSC quiescence and self-renewal pre-

Conflict of interest: The authors have declared that no conflict of interest exists. Citation for this article: J Clin Invest. 2010;120(6):2058-2069. doi:10.1172/JCI42032. dominantly through $\mathrm{Mpl}$, by negatively regulating JAK2 activation in response to Tpo. Biochemical experiments reveal that the Lnk $\mathrm{SH} 2$ domain directly binds to the phosphorylated tyrosine residues 813 (Y813) in JAK2 following Tpo stimulation (8). Therefore, Lnk controls hematopoietic stem and progenitor cell (HSPC) selfrenewal in part through direct interactions with $\mathrm{Mpl} / \mathrm{JAK} 2$ (8).

The amplitude and duration of cytokine receptor signaling are highly regulated, and abnormally sustained signaling can promote leukemic transformation. Myeloproliferative diseases (MPDs) constitute a group of stem cell-derived clonal diseases that include chronic myeloid leukemia (CML), polycythemia vera $(\mathrm{PV})$, essential thrombocythemia (ET), and myelofibrosis (MF). MPDs result from excessive proliferation of one or more myeloid/ erythroid lineage cells (12). Many MPDs can be attributed to constitutive activation of signal transduction pathways (13). CML was the first in which a chromosomal translocation was identified that fused the $B C R$ and $A B L$ genes, leading to a constitutive active ABL tyrosine kinase. JAK2 dysregulation has also been implicated in several hematological malignancies. Abnormal activation of JAK2 by a chromosomal translocation, resulting in its fusion to TEL transcription factor, was shown to be associated with multiple hematologic malignancies, including atypical CML $(14,15)$. Recently, the V617F mutation in JAK2 has also been observed at high frequencies in several MPDs (>90\% PV and approximately $50 \% \mathrm{ET}$ and MF) (16-18).

When overexpressed in BaF3 cells, Lnk inhibits JAK2V617Fmediated proliferation $(19,20)$. However, the role of Lnk in hematologic malignancies in vivo has not been examined. Many regulators of hematopoiesis and HSC self-renewal, such as Bmi-1, PTEN, HoxB4, Gfi-1, and MLL, are also involved in leukemogenesis (21-25). We therefore hypothesized that Lnk is a potential tumor 
A

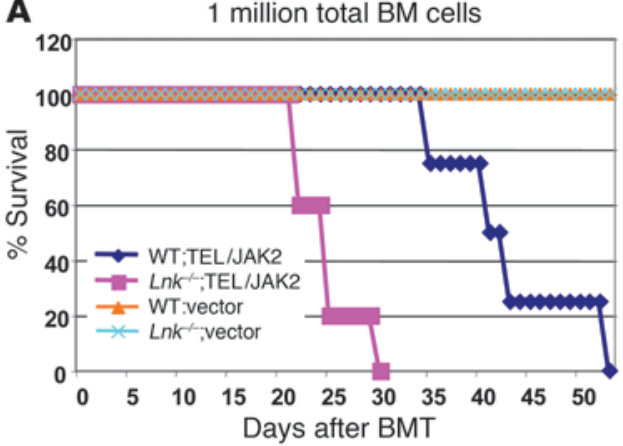

B

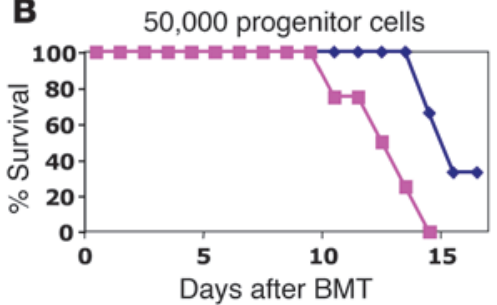

30,000 progenitor cells

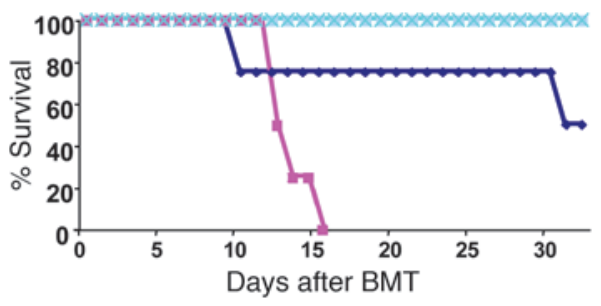

10,000 progenitor cells

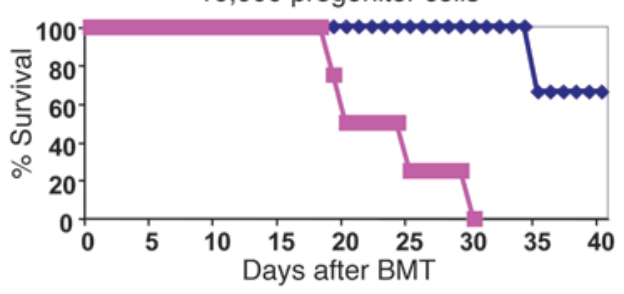

suppressor and that Lnk-deficient HSPCs with augmented JAK2 activity are more susceptible to oncogenic transformation.

Here we examined the role of Lnk in MPD development. We found that loss of Lnk accelerates and exacerbates TEL/JAK2and JAK2V617F-induced MPD in mouse transplant models. Lnk deficiency enhanced cytokine-independent signaling, thereby augmenting the ability of oncogenic JAK2 alleles to expand myeloid progenitors in vitro and in vivo. In addition, a mutant form of JAK2V617F that was unable to interact with Lnk conferred increased myeloid expansion and accelerated MF in the second stage of the disease when compared with JAK2V617F capable of binding Lnk. Hence, we identified Lnk as a physiological negative regulator that may constrain leukemic transformation conferred by oncogenic JAK2. Notably our data suggest that Lnk restrains the expansion of HSPCs, since Lnk deficiency also cooperates with the BCR/ABL oncogene that does not bind Lnk and does not directly rely on JAK2 when initiating MPDs. Lastly, we show that aged $L n k^{-/-}$mice develop a CML-like MPD phenotype, with an abnormal expansion of myeloid cells in the blood, BM, and spleen.

\section{Figure 1}

Lnk deficiency exacerbates MPD development initiated by TEL/JAK2 in mice. (A) WT and $L n k^{-1-}$ BM cells were infected with retroviruses encoding either MIG vector alone or TEL/JAK2, and 1 million total BM cells were transplanted into each irradiated host animal. Kaplan-Meier survival analysis of transplanted mice is shown. $P<0.05$, long-rank test comparing WT;TEL/JAK2 and $L n k^{-1-}$;TEL/JAK2 groups. $n=5$. (B) Lnk deficiency exacerbates CML development initiated by TEL/JAK2 in mice when defined numbers of purified progenitors were transplanted into the hosts. Magnetic bead-enriched Lin- progenitor cells from WT and $L n k^{-/-}$mice were infected with retroviruses, and equal numbers of WT and $\mathrm{Lnk}^{-1-}$ progenitors were transplanted into irradiated host animals. Kaplan-Meier survival analysis of 3 transplants with different progenitor numbers (left, 50,000 progenitor cells; middle, 30,000 progenitor cells; right, 10,000 progenitor cells) is shown. $n=5$ in each group of each transplant.

Thus, our data suggests that Lnk is a bona fide suppressor of MPD in mice and may play a broader role in hematologic malignancies.

\section{Results}

Loss of Lnk enhances and accelerates MPD/CML development in mice. The vast expansion and superior repopulating capability of $L n k^{-/-}$ HSCs $(7,8)$ prompted us to study whether Lnk functions as a potential tumor suppressor by limiting HSPC expansion. To test this, we first determined whether loss of Lnk synergizes with oncogenic JAK2, in causing MPDs in mice.

We isolated 5-fluorouracil-enriched (5-FU-enriched) BM cells from both WT and $L n k^{-/-}$mice and infected them with either murine stem cell vector-internal ribosomal entry site-GFP (MIG, otherwise known as MSCV-IRES-GFP) vector alone or MIG-TEL/ JAK2. When 1 million BM cells were transplanted per lethally irradiated mouse, $L n k^{-/-}$;TEL/JAK2 cells elicited an accelerated and exacerbated CML in the host animals, compared with that of WT;TEL/JAK2 cells (Figures 1, 2, 3). While mice transplanted with WT;TEL/JAK2 cells survived well into the second month, mice transplanted with $\mathrm{Lnk}^{-/-}$;TEL/JAK2 cells all succumbed to CML within the first 4 weeks (Figure 1A).

Since $L n k^{-/-}$mice have 2 -fold elevated CFU-GM progenitors in the BM (ref. 6 and unpublished observations), we examined whether the enhanced CML in mice transplanted with Lnk $^{-/-}$;TEL/ JAK2 cells is intrinsic to Lnk-deficient cells or because a greater number of transduced progenitors were transplanted. To examine this, we transplanted equal numbers of purified, infected, lineage negative $\left(\mathrm{Lin}^{-}\right)$progenitor cells from WT or $\mathrm{Lnk}^{-/-}$mice into irradiated hosts, along with 200,000 freshly isolated WT competitor BM cells (Figure 1B). Lin ${ }^{-}$progenitors from both WT and $\mathrm{Lnk}^{-/-}$ mice were transduced with similar efficiencies (Supplemental Figure 1; supplemental material available online with this article; doi:10.1172/JCI42032DS1). At each cell dosage, TEL/JAK2-transduced $L n k^{-/-}$progenitors conferred reduced survival and accelerated CML compared with TEL/JAK2-transduced WT progenitors (Figure 1B). Thus, Lnk deficiency facilitates MPD development initiated by TEL/JAK2 in mice.

Loss of Lnk leads to exacerbated neutrophilia, with an expansion of the immature myeloid compartment in the BM and spleen. To determine the type of disease that the transplanted mice succumbed to, we examined their hematologic phenotypes. Two to three weeks after transplantation, the wbc counts in mice transplanted with Lnk ${ }^{-/-} ;$TEL/JAK2 BM cells were increased more than 5-fold, com- 
A

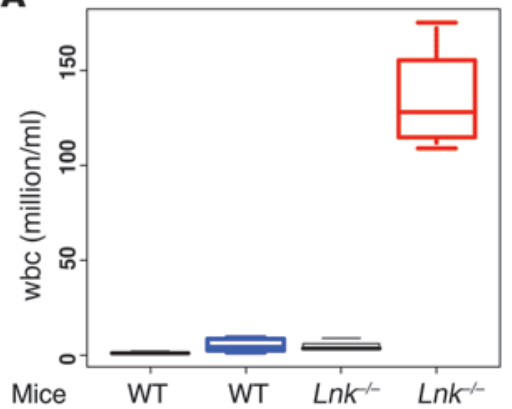

Virus Vector TEL/JAK2 Vector TEL/JAK2

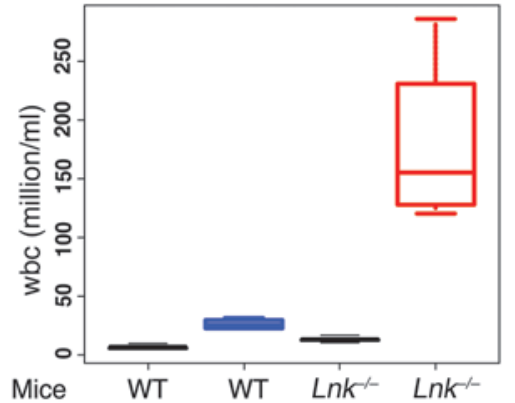

Virus Vector TEL/JAK2 Vector TEL/JAK2
B

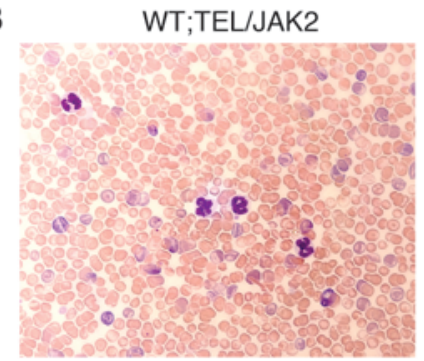

D
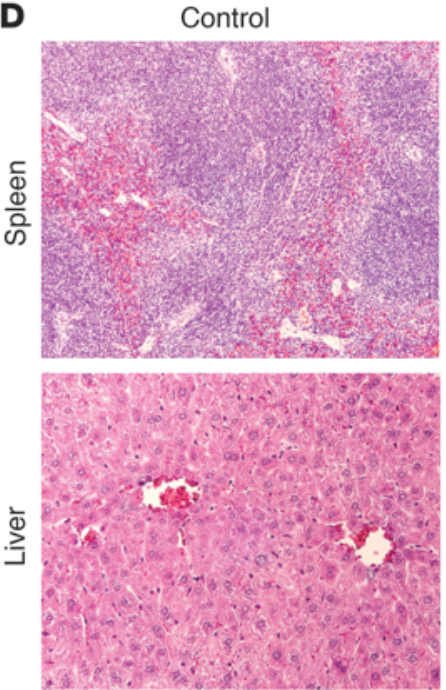

Lnk';'TEL/JAK2
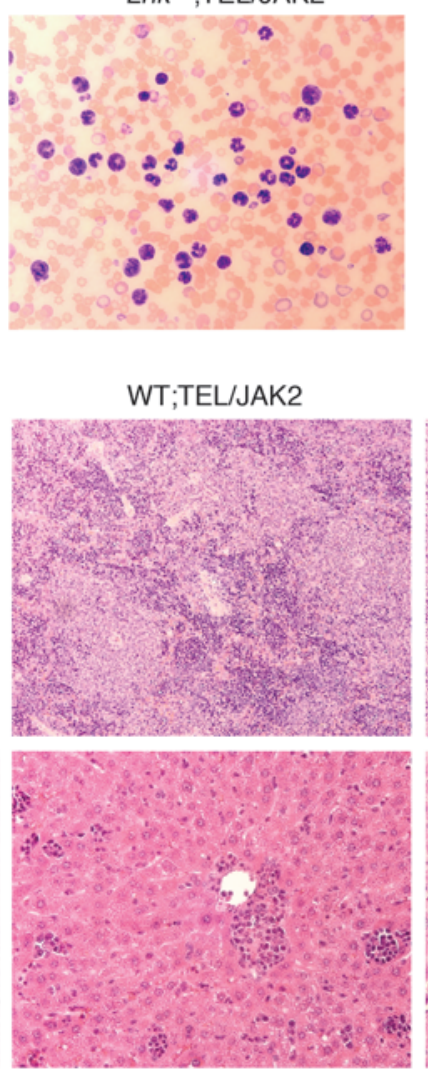

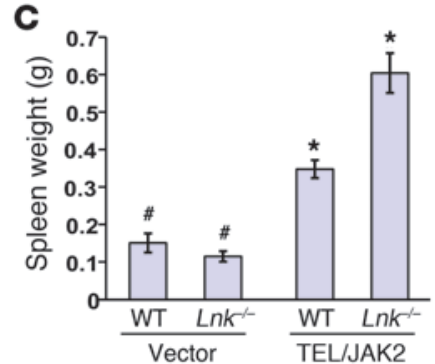

Lnk'-;TEL/JAK2
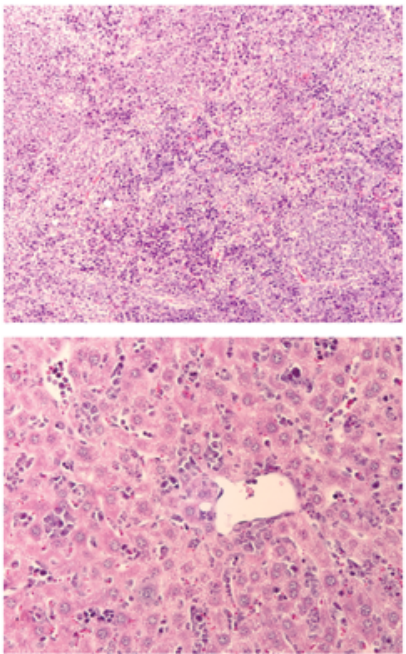

Figure 2

Lnk deficiency leads to an exacerbated neutrophilia and neutrophil infiltration into multiple organs. (A) The box plot shows wbc measured 2-3 weeks after the transplant (left) and in moribund animals (right). The top and bottom ends of the boxes define the 75th and 25th percentiles, the horizontal lines indicate the medians, and the error bars define the 5th and 95th percentiles. $n=5$. $P<0.01$ comparing WT;TEL/JAK2 and $\mathrm{Lnk}^{-1-} ; \mathrm{TEL} / \mathrm{JAK} 2$ groups. (B) Wright-Giemsa staining of blood smears from WT;TEL/JAK2 and Lnk $^{-1-} ;$ TEL/JAK2 mice shows massively increased numbers of myeloid cells at $2-3$ weeks. Original magnification, $\times 400$. (C) Spleen weight of transplanted mice (average \pm SEM). ${ }^{\#} P=\mathrm{NS}$; ${ }^{*} P<0.005$. $n=4-6$. (D) H\&E staining of tissue sections. Original magnification, $\times 100$ (top row, spleen); ×200 (bottom row, liver). pared with mice transplanted with WT;TEL/JAK2 BM cells (Figure $2 \mathrm{~A}$, left). This increase was also found in moribund animals (Figure 2A, right). Blood smears confirmed that Lnk $^{-/-}$;TEL/JAK2 mice exhibited more severe neutrophilia than WT;TEL/JAK2 mice (Figure 2B). Neutrophilia correlated with increased spleen weight (Figure 2C) and neutrophil infiltration into multiple organs (Figure 2D) in both WT;TEL/JAK2 and $L n k^{-/-}$;TEL/JAK2 mice, recapitulating major clinical features of CML patients (26). The disruption of splenic structure and increase of spleen weight were greater in $L n k^{-/-}$; TEL/JAK2 mice, and neutrophil infiltration into the liver was more diffuse and dramatic (Figure 2D). Therefore, Lnk deficiency exacerbates neutrophilia and neutrophil infiltration into multiple organs.

To extend our morphologic examinations of peripheral blood, we performed flow cytometric analysis, using myeloid and lymphoid markers. We found that almost all $\mathrm{GFP}^{+}$cells in the blood are Gr-1/Mac-1 $1^{+}$myeloid cells, in both WT;TEL/JAK2 and $L n k^{-/-}$ ;TEL/JAK2 mice (Figure 3, A and B). Importantly, Lnk ${ }^{--}$;TEL/JAK2 mice exhibited a marked expansion of myeloid cells, with over $80 \%$ of blood cells being donor derived $\left(\mathrm{GFP}^{+}\right.$and $\left.\mathrm{Gr}-1 / \mathrm{Mac}-1^{+}\right)$; in comparison, fewer than $50 \%$ of blood cells were donor derived in WT;TEL/JAK2 mice (Figure 3A). In contrast, mice transplanted with vector-infected WT or $L n k^{-/-}$cells showed reconstitution of multiple lineages, including Gr- $1 / \mathrm{Mac}-1^{+}$myeloid, $\mathrm{CD} 4 / \mathrm{CD}^{+} \mathrm{T}$, and $\mathrm{B} 220^{+} \mathrm{B}$ cells (Figure 3, C and D).

We next examined lineage reconstitution in the $\mathrm{BM}$ and spleens of the transplanted mice. Strikingly, Lnk $k^{-/} ;$TEL/JAK2 BM and spleens exhibited an increased accumulation of $\mathrm{Gr}-1^{\text {lo }}$ immature myeloid cells in comparison with Gr-1 ${ }^{\text {hi }}$ mature myeloid cells in WT;TEL/ JAK2 mice (Figure 3, E and G). GFP ${ }^{+}$-infected cells, gated for Mac-1 and $\mathrm{Gr}-1$, revealed an expansion of immature myeloid cells relative to mature ones $\left(\mathrm{Mac}-1^{+} \mathrm{Gr}-1^{\text {lo }}\right.$ versus $\mathrm{Mac}-1^{+} \mathrm{Gr}-1$ hi; ref. 27) 
A
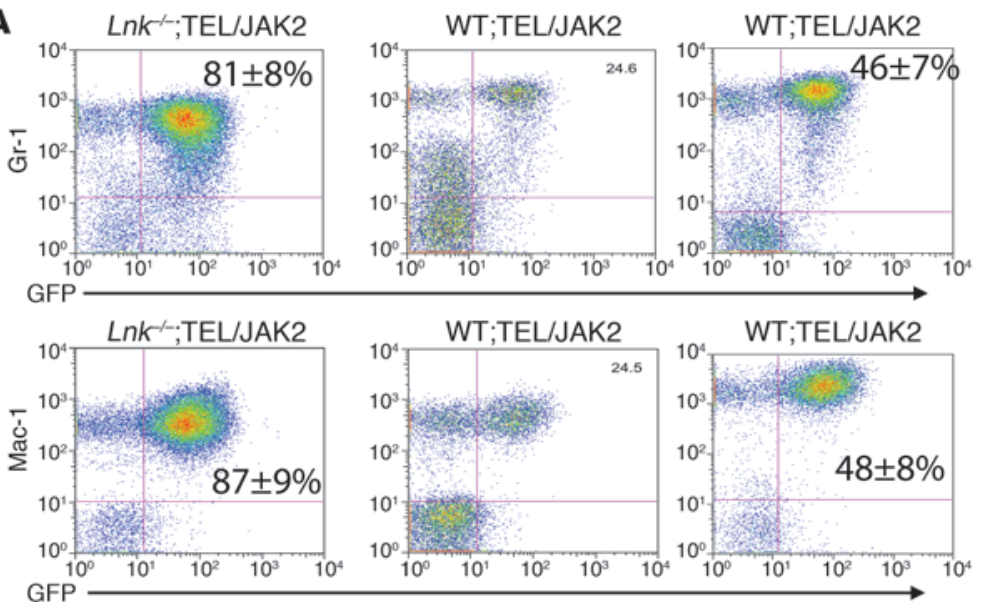
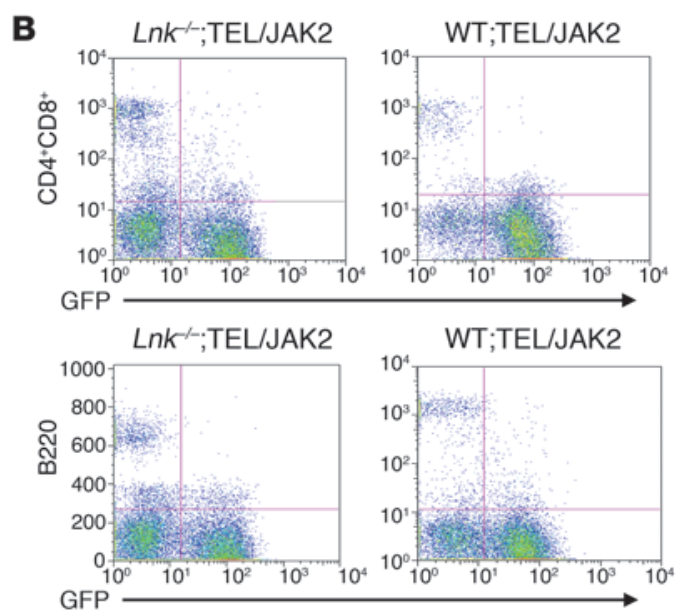

D
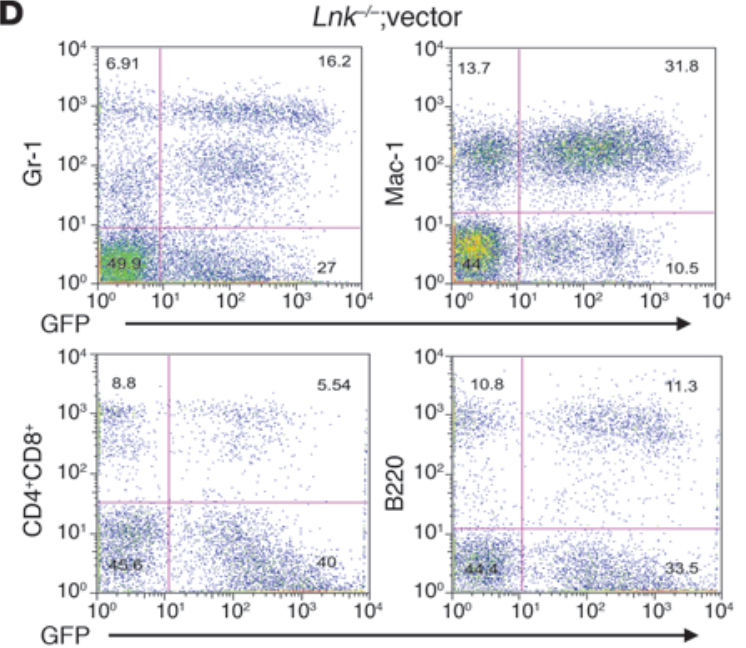

$\mathbf{F}$

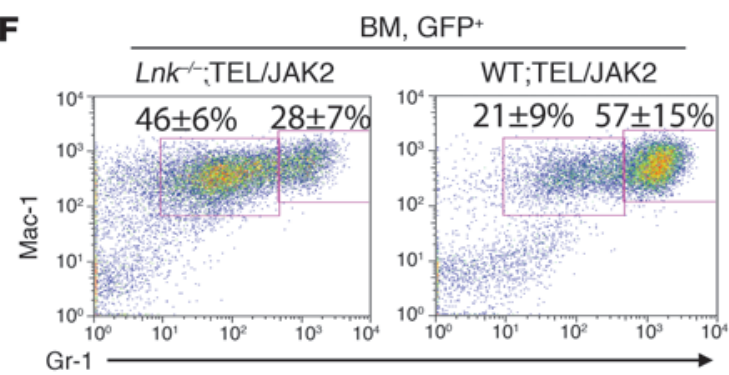

\section{Figure 3}

Lnk deficiency leads to an exacerbated neutrophilia, with an expansion of immature myeloid cells in the BM and spleen. Peripheral blood and tissue lineage chimerism analysis after transplantation was performed using flow cytometry. (A) Myeloid chimerism of Lnk $^{-1-}$;TEL/JAK2 (left) and WT;TEL/JAK2 (middle) mice 2 weeks after BMT and moribund WT;TEL/JAK2 mice (right). Percentages (average \pm SEM) of GFP + myeloid cells are shown. $P<0.001$ comparing WT;TEL/JAK2 and $\mathrm{Lnk}^{-1-}$;TEL/JAK2 groups. (B) Lymphoid chimerism of Lnk $^{-1-}$;TEL/JAK2 and WT;TEL/JAK2 mice. (C and D) Blood lineage chimerism of WT; vector and $L n k^{-1-}$; vector mice 3-4 weeks after BMT. Representative percentages of lineage distributions versus GFP expression are indicated. (E-G) Myeloid composition in the BM (E and $\mathbf{F})$ and spleen $(\mathbf{G})$ of WT;TEL/JAK2 and $\mathrm{Lnk}^{-1-}$;TEL/JAK2 mice. (E) The percentage of GFP $+\mathrm{Gr}-1^{\text {hi }}$ and $\mathrm{GFP}+\mathrm{Gr}-1^{10}$ $\mathrm{BM}$ cells is quantified (average $\pm \mathrm{SEM}$ ). $n=8 . P<0.01$. (F) Mac-1 and Gr-1 expression of $\mathrm{GFP}^{+}$-gated BM cells. The percentage of Mac- $1^{+} \mathrm{Gr}-1^{\text {hi }}$ mature and Mac- $1^{+} \mathrm{Gr}-1^{\mathrm{lo}}$ immature myeloid cells is quantified (average $\pm \mathrm{SEM}$ ). $n=8 . P<0.01$. (G) The percentage of GFP $+\mathrm{Gr}-1^{10}$ splenic cells (average \pm SEM). $n=6 . P<0.05$. 
A
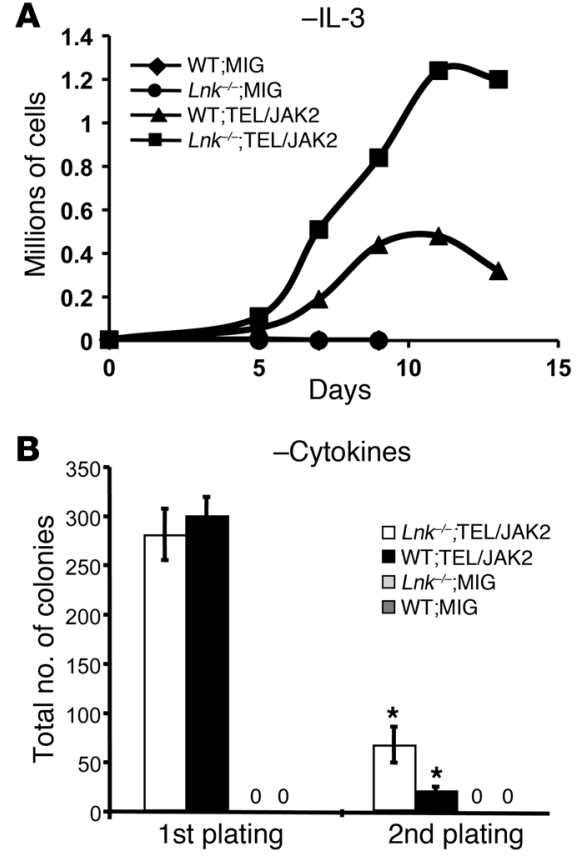
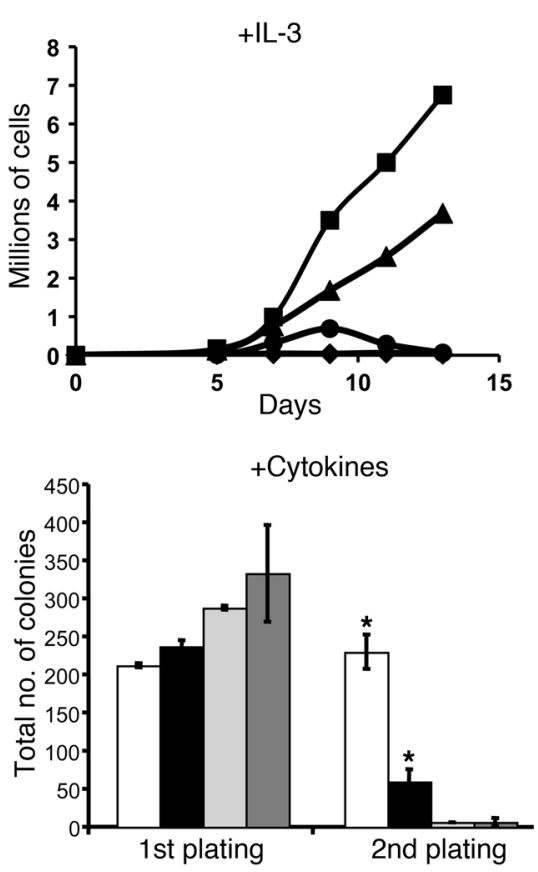

\section{Figure 4}

Lnk deficiency leads to an enhanced progenitor cell proliferation and self-renewal in vitro. Lin- progenitor cells from WT and $L n k^{-/-}$mice were infected with retroviruses encoding either vector alone or TEL/JAK2 and subsequently sorted for GFP positivity. (A) Purified GFP+Lin ${ }^{-}$ progenitors were cultured in the absence of cytokine (left) and in the presence of WEHI supernatant containing IL-3 (right). Live cells were enumerated every $2-3$ days, and representative growth curves are shown. The graphs show representatives of 5 independent experiments. (B) Purified GFP+Lin- progenitors were plated in cytokine-free (M3234) or cytokinerich (M3434) methylcellulose media (StemCell Technologies Inc.). Eight to ten days later, colonies were enumerated, replated, and scored again another $8-10$ days later. The total colony numbers of 1 st and 2 nd platings are shown (average $\pm \mathrm{SD}$ ). ${ }^{*} P<0.01 . n=4-5$. in $L n k^{-/-} ; \mathrm{TEL} / \mathrm{JAK} 2 \mathrm{BM}$ (Figure 3F). This flow cytometric analysis is consistent with BM cytological analyses (Supplemental Figure 2). Thus, Lnk deficiency leads to an exacerbated neutrophilia, with an expansion of immature myeloid cells in the BM and spleen.

Loss of Lnk leads to enhanced progenitor cell expansion in vitro and in vivo and increased cytokine signaling. The marked increase in the number of myeloid cells in Lnk $k^{-/-}$;TEL/JAK2-transduced mice suggests that Lnk deficiency may facilitate myeloid progenitor cell expansion. We thus studied the proliferation and self-renewal of WT and $L n k^{-/-}$progenitor cells. Lin ${ }^{-}$progenitor cells from WT and $L_{n k^{-/-}}$BM cells were infected with retroviruses encoding either MIG alone or TEL/JAK2, and purified $\mathrm{GFP}^{+} \mathrm{Lin}^{-}$progenitor cells were cultured either in the absence or presence of cytokines (Figure 4). In liquid culture, $L n k^{-/-}$; TEL/JAK2 progenitor cells showed enhanced proliferation, both in the absence (Figure 4A) and presence of IL-3 (Figure 4A). When plated in semisolid methylcellulose culture, $L n k^{-/-} ;$TEL/JAK2 progenitor cells gave rise to similar numbers of colonies as WT;TEL/JAK2 cells in cytokine-free conditions; however, the former generated significantly more colonies in secondary platings (Figure 4B). Vector-infected WT and $\mathrm{Lnk}^{-/-}$progenitors failed to generate any colonies, indicating that Lnk deficiency does not result in cytokine-independent growth. In cytokine-replete conditions, vector- and TEL/JAK2-transduced WT and $\mathrm{Lnk}^{-/-}$cells generated high numbers of colonies in the first plating. However, $L n k^{-/-} ; \mathrm{TEL} / \mathrm{JAK} 2$ progenitor cells gave rise to significantly more colonies than WT;TEL/JAK2 progenitors in the secondary platings, while vector-infected WT or $L n k^{-/-}$progenitors failed to re-plate (Figure 4B). These data suggest that loss of Lnk promotes progenitor cell proliferation and self-renewal in vitro.

The enhanced proliferation and self-renewal of $\mathrm{Lnk}^{-/-}$;TEL/JAK2 progenitor cells in vitro prompted us to further assess myeloid progenitor expansion in vivo. We isolated BM and spleen cells 2 weeks after transplantation and quantified progenitor numbers in methylcellulose culture assays. We found that TEL/JAK2 expression promoted the expansion of WT progenitors 1.7- and 1.5-fold in the spleen and BM, respectively, when compared with controls. In contrast, TEL/JAK2 caused a 2.5- and 2.6-fold expansion of $L n k^{-/-}$ progenitors in the spleen and $\mathrm{BM}$, respectively (Figure $5 \mathrm{~A}$ ). Thus, Lnk deficiency predisposed progenitors to TEL/JAK2- induced expansion in vivo.

Lnk limits basal level JAK/Stat signaling. We determined whether JAK/Stat signaling was enhanced by isolating BM cells from mice reconstituted with WT or $L n k^{-/-}$cells expressing MIG or TEL/ JAK2, cytokine starving them, then further incubating them in the absence or presence of Tpo or IL-3 for 10 minutes. We failed to detect signals stimulated with $10 \mathrm{ng} / \mathrm{ml} \mathrm{Tpo}$, probably due to limited Mpl expression. Mpl is expressed in LSK cells but only in a small fraction, if at all, of progenitor cells or committed myeloid cells $(8,28)$. In contrast, $10 \mathrm{ng} / \mathrm{ml}$ IL-3 administration robustly stimulated all major signaling pathways (Stat5, Akt, and ERK) in both WT and $L n k^{-/}$groups expressing TEL/JAK2 or vector alone. Importantly, we observed cytokine-independent activation of

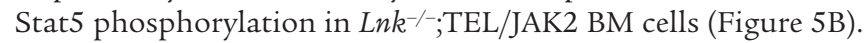
This indicates that loss of Lnk augments constitutive active JAK2/ Stat signaling in the transplanted mice expressing TEL/JAK2.

WT Lnk but not SH2-deficient Lnk inhibits MPD/CML development. We previously demonstrated that the Lnk SH2 domain is essential for its inhibitory functions in primary hematopoietic cells $(10,29)$ and is critical for JAK2 binding (8). In order to dissect the importance of the Lnk SH2 domain in oncogenic JAK2-induced CML/ MPD, we performed "rescue" experiments, in which we transduced $\mathrm{Lin}^{-}$progenitor cells from $\mathrm{Lnk^{-/- }}$ mice with TEL/JAK2, along with either WT Lnk or a mutant version with an R364E substitution in the SH2 domain (LnkRE). Coexpression of TEL/JAK2 and Lnk was accomplished by using MSCV-TEL/JAK2-IRES-GFP/Lnk expression vectors (Figure 6A). Following transplantation into host animals, we monitored onset and kinetics of MPD development. Lnk $k^{-/} ;$TEL/JAK2-Lnk conferred CML at a much slower rate when compared with $L n k^{-/-} ;$TEL/JAK2-transduced cells (80 days vs. 30 days) (Figure 6 and Supplemental Figure 3). In contrast, $L^{-1 /-}$; 

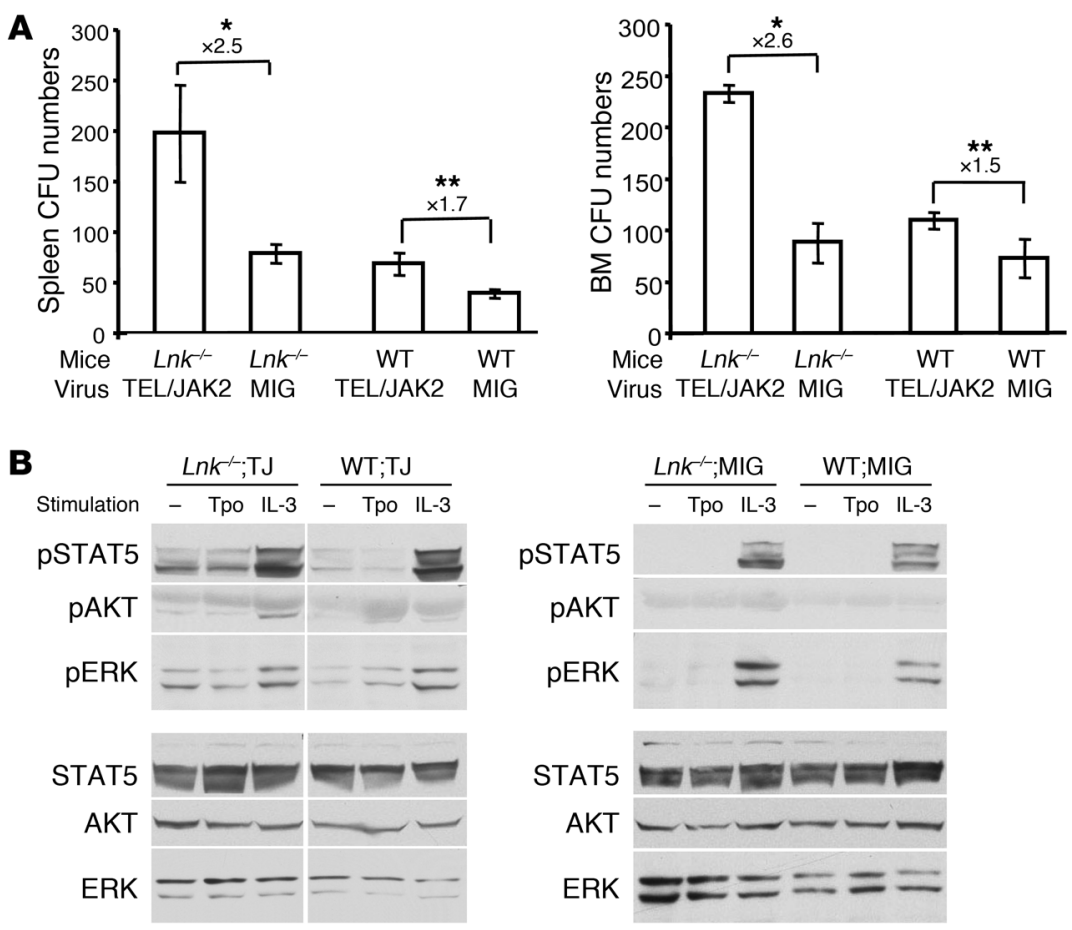

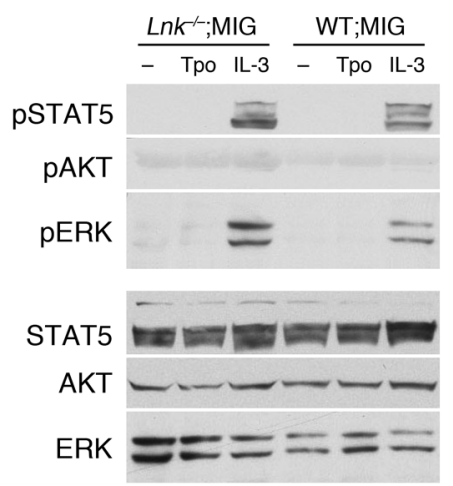

\section{Figure 5}

Lnk deficiency increases the ability of TEL/JAK2 to expand progenitors and enhances cytokine signaling. Two weeks after BMT, spleen and BM cells from mice reconstituted with WT and $L n k^{-1-}$ cells expressing MIG or TEL/JAK2 (TJ) were isolated. (A) Spleen and BM cells were plated on M3434 methylcellulose media, and CFU progenitors were quantified at days 8-10. Fold differences (average \pm SEM) between indicated groups are shown above the bars. ${ }^{\star} P<0.01$; ${ }^{\star \star} P<0.05 . n=4$. (B) BM cells were starved, then either left unstimulated or stimulated with either $10 \mathrm{ng} / \mathrm{ml}$ of Tpo or IL-3 for 10 minutes before lysis. Protein lysates were subjected to Western blot analysis. Representative results of 4 independent experiments are shown.
TEL/JAK2-LnkRE conferred CML in a similar fashion to that of Lnk ${ }^{-/-}$;TEL/JAK2 (Figure 6). We conclude that Lnk constrains TEL/JAK2-induced CML in a cell intrinsic manner, at least in part, through its $\mathrm{SH} 2$ domain.

Lnk deficiency accelerates PV in mice. The V617F mutation in JAK2 has been found at high frequency in MPD patients (16-18). We previously observed that JAK2V617F retains Lnk binding ability, suggesting that Lnk could modulate MPD development resulting from the JAK2V617F mutation. To examine the role of Lnk in MPD development, we isolated BM cells from WT and $L n k^{-/-}$ mice after 5-FU treatment, infected the cells with either MIG or MIG-JAK2V617F, and subsequently transplanted them into lethally irradiated mice. Mice transplanted with Lnk $^{-/-}$;JAK2V617F cells developed PV much earlier than those transplanted with

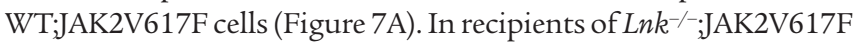
cells, the hematocrits were elevated as early as 3 weeks after transplantation. In contrast, WT;JAK2V617F-transplanted mice developed PV around 5 weeks, as previously reported $(30,31)$ (Figure 7A). Thus, our data suggest that Lnk deficiency accelerates the onset of JAKV617F-induced PV in mice.

Lnk restricts myeloid expansion and $M F$ development in mice. The JAK2V617F construct maintains Lnk binding capability in hematopoietic cells (8), and a physical interaction between Lnk and the JAK2V617F oncogene may directly contribute to restraining $\mathrm{MPD}$ in mice. We thus introduced the Y813F mutation into the JAK2VF construct to ablate Lnk binding (Supplemental Figure 4) and determined whether this mutation enhances the ability of JAK2V617F to cause MPD.

Lin $^{-}$progenitor cells from WT and $L n k^{-/-}$mice were infected with retroviruses producing JAK2wt, JAK2V617F, and JAK2V617F/ Y813F and transplanted into irradiated recipients. JAK2V617F expression in WT C57/B6 mice primarily confers PV, characterized by moderately increased circulating myeloid and platelet counts $(30,31)$. In contrast, JAK2V617F expression in $\mathrm{Lnk}^{-/-}$cells triggered a marked expansion of neutrophils in the peripheral blood. Importantly, mice transplanted with WT cells expressing JAK2V617F/ Y813F had increased neutrophil counts when compared with WT;JAK2V617F mice (Figure 7B). WT;JAK2Y813F and $\mathrm{Lnk}^{-/-}$ ;JAK2Y813F mice displayed similar hematocrits and wbc counts to those transplanted with WT or $L n k^{-/-}$cells expressing WT JAK2 (data not shown). Together, these data indicate that Lnk deficiency enhances JAKV617F-induced myeloid expansion in mice.

We further examined the progression of the disease into the MF phase. WT;JAK2V617F mice progressed into MF 4 months after transplantation, as previously reported (31). WT;JAK2V617F/ Y813F mice progressed into MF (Figure 7C) and anemia (data not shown) faster than WT;JAK2V617F mice, while Lnk $^{-/-}$;JAK2V617F and $L n k^{-/-}$;JAK2V617F/Y813F mice exhibited similar MF status. Specifically, we found that $\mathrm{Lnk}^{-/-}$;JAK2V617F-engrafted mice displayed more severe MF than WT;JAK2V617F-engrafted mice, as evidenced by reticulin staining of the BM and spleen and enhanced myeloid infiltration in the liver at 2 months (Figure $7 \mathrm{C}$ ). Importantly, WT;JAK2V617F/Y813F mice developed more severe and early onset of MF than WT;JAK2VF mice (Figure 7C). Therefore, our data suggest that Lnk restricts myeloid expansion and the onset of MF in JAK2V617F-reconstituted mice, in part by directly attenuating the JAK2V617F oncogene.

The ability of Lnk to modulate MPD development is not restricted to oncogenic JAK2. Our results above show that Lnk acts in part by directly inhibiting exogenous JAK2V617F oncogene. However, WT cells expressing JAK2V617F/Y813F caused a less severe MPD phenotype than $L n k^{-/-}$;JAK2V617F cells, suggesting Lnk might additionally function by inhibiting endogenous signaling pathways in HSPCs. If true, this would imply that Lnk deficiency might cooperate with oncogenes that do not directly rely on JAK2 or bind Lnk. To test this hypothesis, we examined the role of Lnk in modulating CML development caused by BCR/ABL, which does not directly interact with Lnk (32). We found that $L n k^{-/-} \mathrm{BM}$, transduced with BCR/ 
A

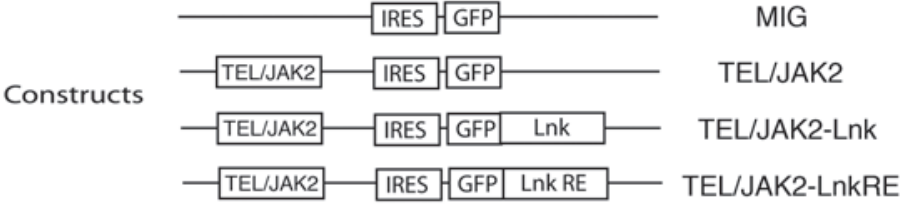
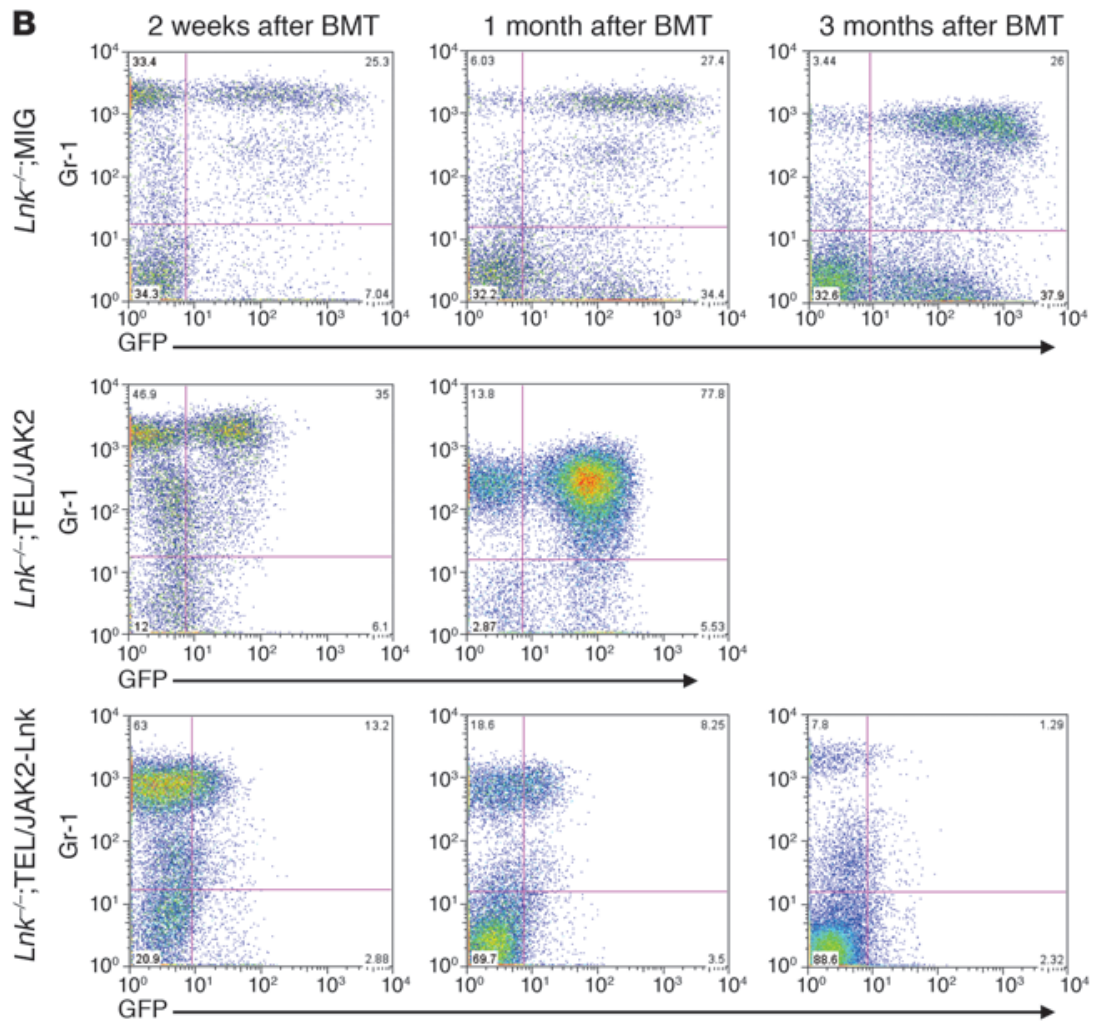

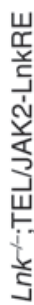

峁
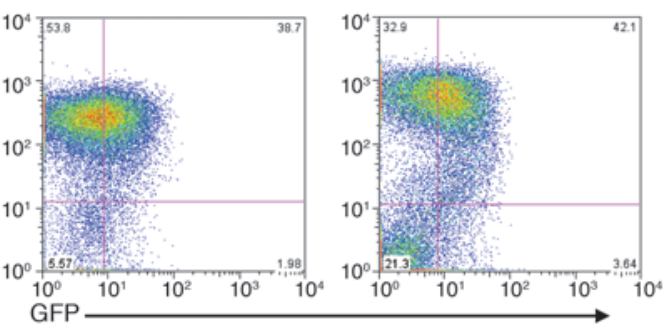

c

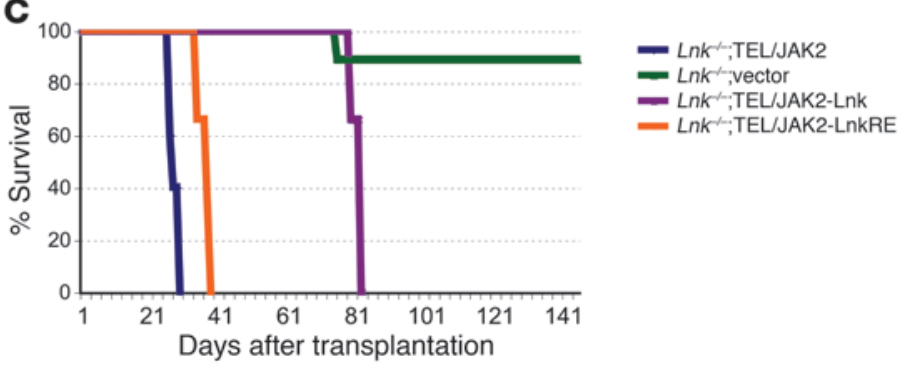

Figure 6

WT Lnk but not SH2- deficient Lnk can rescue CML development from $\mathrm{Lnk}^{-/-}$cells expressing TEL/JAK2. (A) Schematic diagram of retroviral constructs used for infection and transplantation. $\mathrm{Lin}^{-}$progenitor cells from $\mathrm{Lnk}^{-1-}$ mice were infected with retroviruses encoding either MIG, TEL/JAK2, TEL/JAK2-Lnk, or TEL/JAK2-LnkR364E (TEL/ JAK2-LnkRE) and transplanted into irradiated recipients. (B) Myeloid chimerisms of reconstituted mice at indicated time points after transplantation. (C) Survival curves of reconstituted mice.

Aged Lnk-/- mice develop a CML-like MPD. Lnk restricts myeloid expansion in transplanted cells expressing diverse oncogenes. This raised the possibility that $L n k^{-/-}$mice might spontaneously develop MPDs as they age and accumulate genetic mutations or rearrangements that might promote neoplastic transformation. Young Lnk $k^{-/}$mice (2 months of age) exhibit a 3 -fold increase in platelets as well as wbc (ref. 6 and Figure $8 \mathrm{~A}$ ). This is accompanied by a proportionate increase of both myeloid and lymphoid cells in the peripheral blood, BM, and spleen (6), likely resulting from elevated HSPC numbers. However, old $L n k^{-/-}$mice (12-18 months of age) displayed a more dramatic elevation in wbc counts. Moreover, neutrophils and monocytes were expanded disproportionately when compared with other lineages (Figure 8A). Old $\mathrm{Lnk}^{-/-}$mice additionally showed a 5 -fold increase in myeloid cells when compared with young $\mathrm{Lnk}^{-/-}$mice and a 9-fold myeloid expansion when compared with age-matched WT mice. In contrast, lymphocyte counts of old $L n k^{-/-}$mice were only marginally increased (approximately 1.7-fold), while the platelet counts remained unchanged when compared with young $\mathrm{Lnk}^{-/-}$mice (Figure 8A).

Previous work showed that young $\mathrm{Lnk}^{-/-}$mice exhibit a 2-fold increase in spleen weight, due to an expansion of $B$ cells and megakaryocytes $(6,10)$. Aged $L n k^{-/-}$mice displayed a more pronounced splenomegaly, with a disproportionate expansion of myeloid cells, as determined by flow cytometry and histology (Figure 8, B and C). Specifically, mutant spleens exhibited marked extramedullary hematopoiesis, with disruption of the normal splenic architecture, due to infiltration of mature myeloid cells into the red pulp along with increased numbers of megakaryocytes (Figure 8D). In agreement with these findings, flow cytometry revealed a 5 - to 6 -fold increase in the percentage of Mac- $1^{+} \mathrm{Gr}-1^{+}$

$\mathrm{ABL}$, resulted in a more aggressive $\mathrm{CML}$ when compared with WT BM expressing BCR/ABL (Supplemental Figure 5). Two weeks after transplantation, Lnk deficiency markedly increased neutrophilia and myeloid expansion in the BCR/ABL transplant models (Supplemental Figure 5). Thus, the ability of Lnk to modulate MPD development is not restricted to oncogenic JAK2. cells, accompanied by a reduction in B cell percentages. Histological analysis of the BM of aged $\mathrm{Lnk}^{-/-}$mice also revealed increased numbers of granulocytic and monocytic cells (Figure 8D). Finally, periportal cuffing of liver sinusoids with infiltrating myeloid cells was present in the mutant mice (Figure 8E). Taken together, aged $\mathrm{Lnk}^{-/-}$mice developed a CML-like MPD. 
A

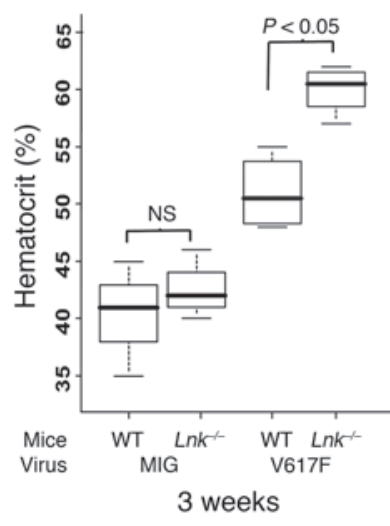

C
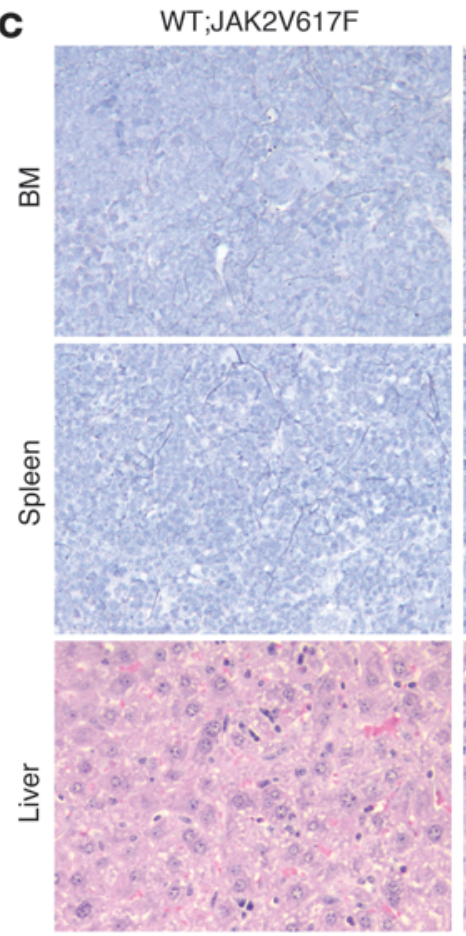

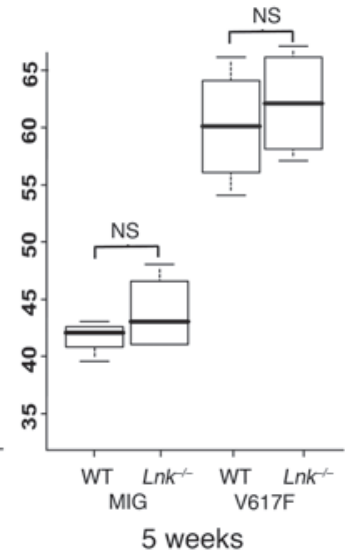

B

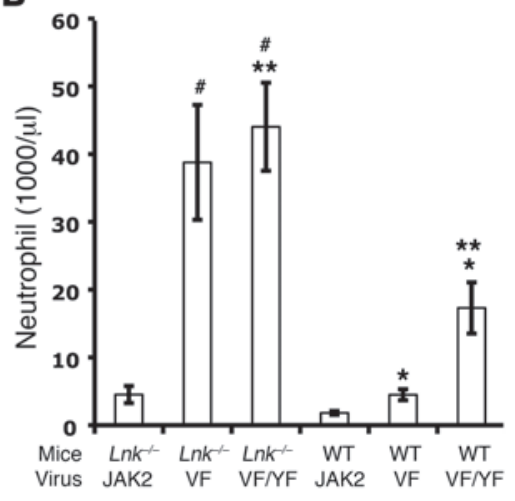

WT;JAK2V617F/Y813F
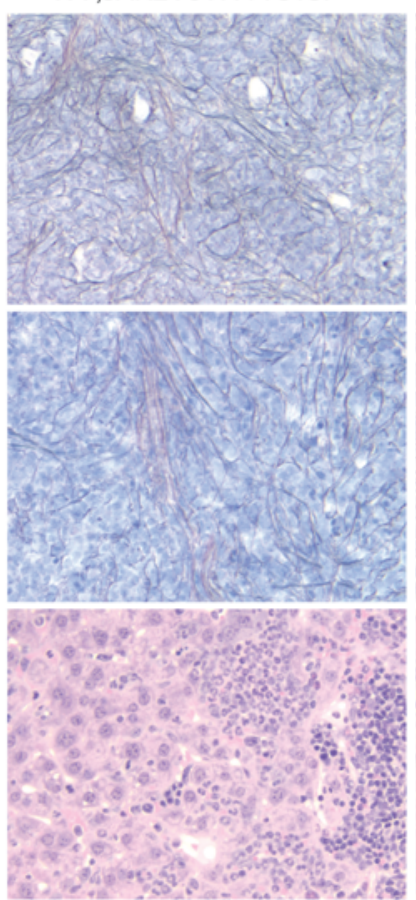

Lnk';JAK2V617F

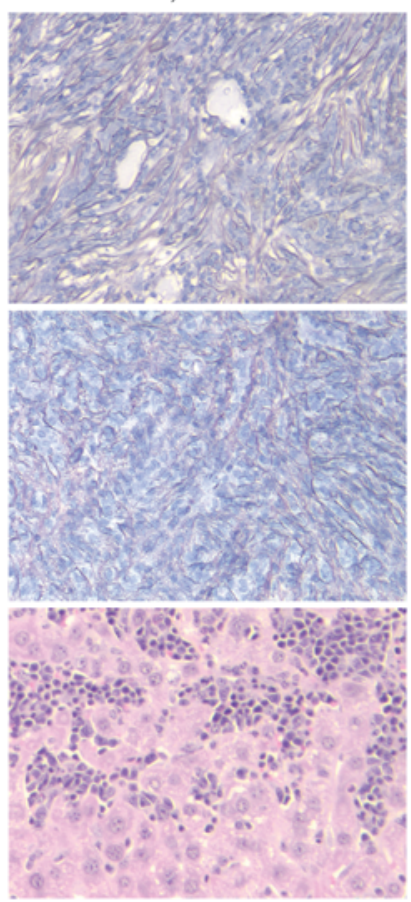

Figure 7

Lnk deficiency accelerates the onset of PV initiated by JAK2V617F and MF progression in mice. (A) The box plot shows hematocrits measured from mice transplanted with WT and $L_{n k^{-1-}}$ cells infected with retroviruses (either MIG vector or JAK2V617F) at week 3 and week 5 . The top and bottom ends of the boxes define the 75th and 25th percentiles, the horizontal lines indicate the medians, and the error bars define the 5 th and 95th percentiles. $n=5$; 2-tailed Student's $t$ tests are shown. (B) Lnk restricts myeloid expansion of JAK2V617F-reconstituted mice. WT and $\mathrm{LnK}^{-1-} \mathrm{BM}$ cells were infected with JAK2wt, JAK2V617F, or JAK2V617F/Y813F viruses and transplanted into irradiated recipients. Neutrophil counts in the peripheral blood were analyzed 2 months after transplant (average \pm SEM). VF, JAK2V617F; VF/YF, JAK2V617F/Y813F. " $P=N S$; ${ }^{*} P<0.005$; ${ }^{\star} P<0.05$. (C) Reticulin stainings are shown in the BM and spleen sections (top and middle panels), and H\&E stainings are shown for the liver sections (lower panel) of transplanted mice. Original magnification, $\times 200$.

\section{Discussion}

Earlier work suggested that Lnk negatively regulates normal HSPC expansion and self-renewal, in part through the Tpo/Mpl/JAK2 signaling pathway $(8,9,11)$. However, the role of Lnk in malignant HSPC expansion has not been studied. When overexpressed in cell lines, Lnk inhibits JAK2V617F-mediated proliferation in BaF3 cells $(19,20)$. However, the role of Lnk in hematologic malignancies in vivo had not been examined.

Here we found that loss of Lnk accelerated and exacerbated oncogenic JAK2-induced MPD development in mice. Lnk deficiency stimulated the expansion of immature myeloid progenitor cells in transplanted mice, as a result of enhanced progenitor proliferation and self-renewal. In addition, Lnk deficiency enhanced cytokine-independent JAK/Stat signaling and conveyed hypersensitivity to oncogenic JAK2-mediated progenitor cell expansion in vivo. Furthermore, our structure-function studies suggest that the Lnk SH2 domain is important for Lnk's inhibitory function during malignant progenitor cell transformation. The fact that Lnk restricts myeloid expansion in the transplant models prompted us to investigate aged $\mathrm{Lnk}^{-/-}$mice that might have accumulated additional mutations that promote neoplastic progression. Indeed, we found that aged $\mathrm{Lnk}^{-/-}$mice spontaneously develop a CML-like MPD. Myeloid cells were markedly expanded in peripheral blood, $\mathrm{BM}$, and spleen and infiltrated into livers in the mutant mice. Notably, the MPD development occurred in the absence of forced expression of oncogenes or BM transplantation (BMT). Therefore, our data establish Lnk as a bona fide suppressor of MPD.

Cytokine binding to its cognate receptor induces activation of JAKs, which in turn triggers a cascade of signaling events (2). These signaling events include a variety of positive mediators, while at the same time, they also initiate multiple negative regulators, such as protein tyrosine phosphatase SHP-1 (33) and the E3 ubiquitin ligase $\mathrm{Cbl}(34,35)$. In fact, gene profiling analysis of IL-6-treated cells identified a large set of cytokine-induced genes that includes the SOCS protein family, which attenuates JAK/Stat signaling (36, 37). Therefore, activation of signaling attenuators is an intrinsic 

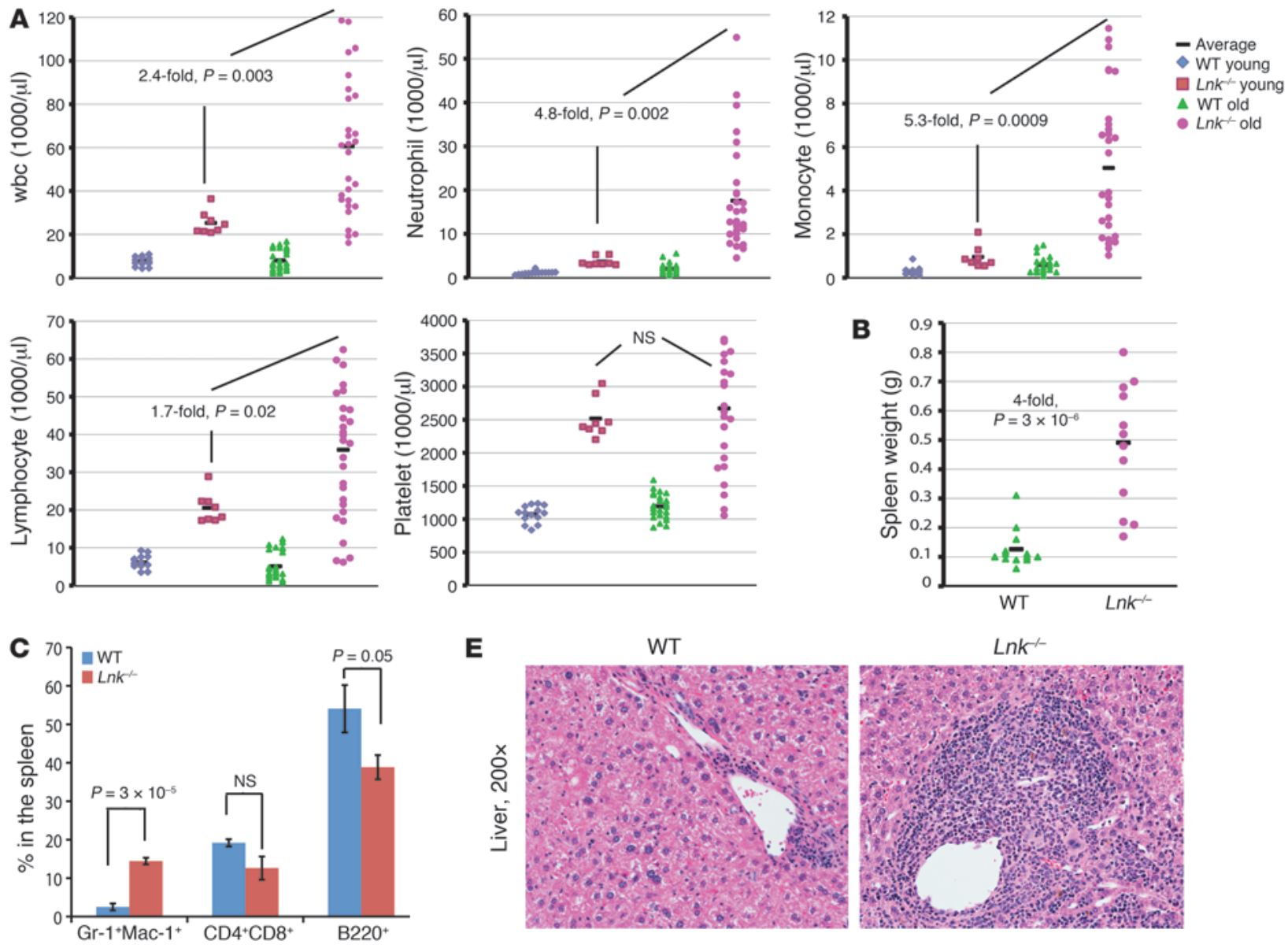

E

WT

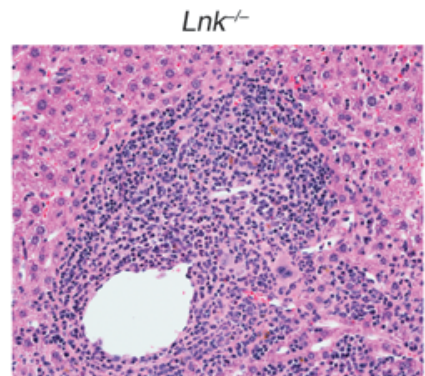

D

BM, 400x
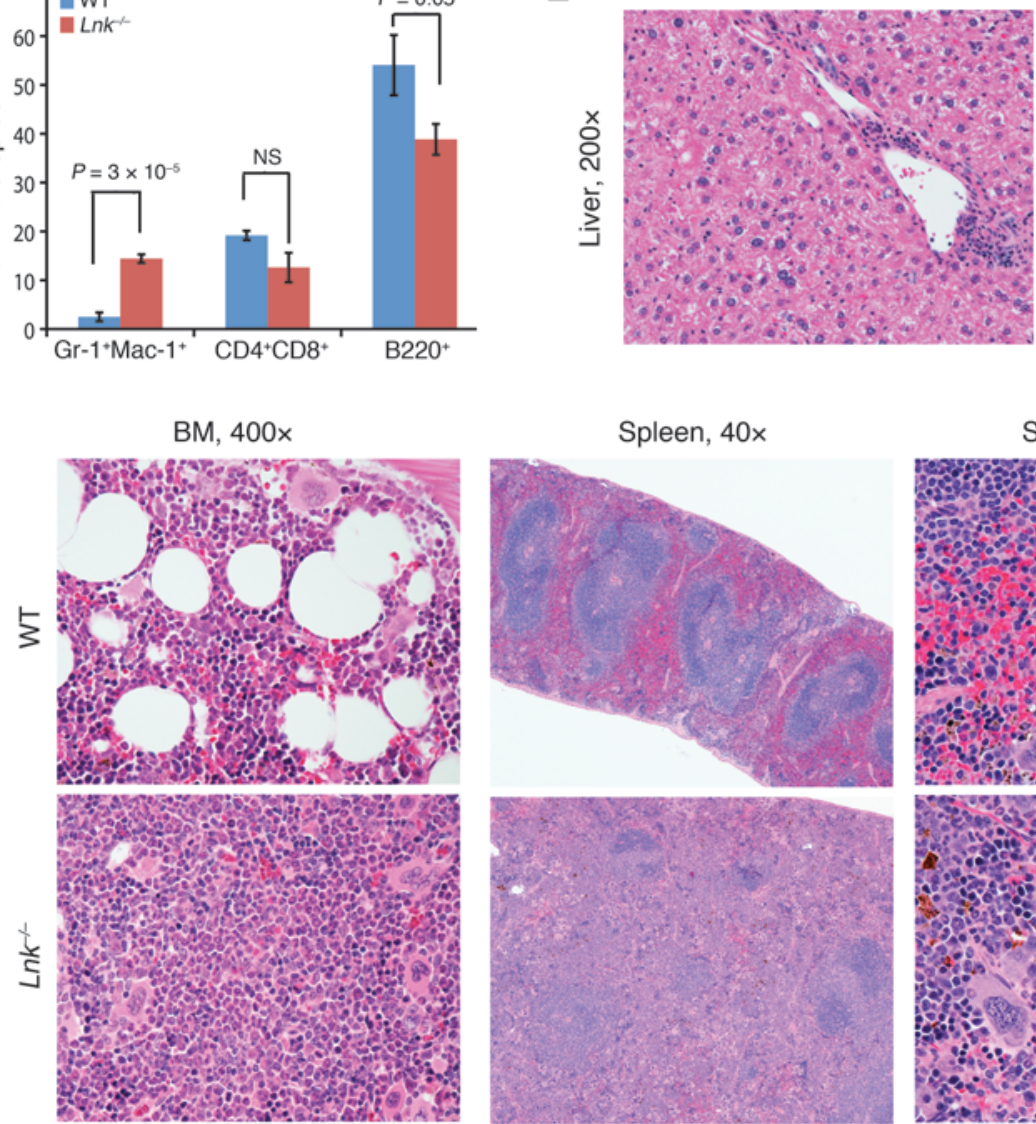

WT old

- Lnk'- old
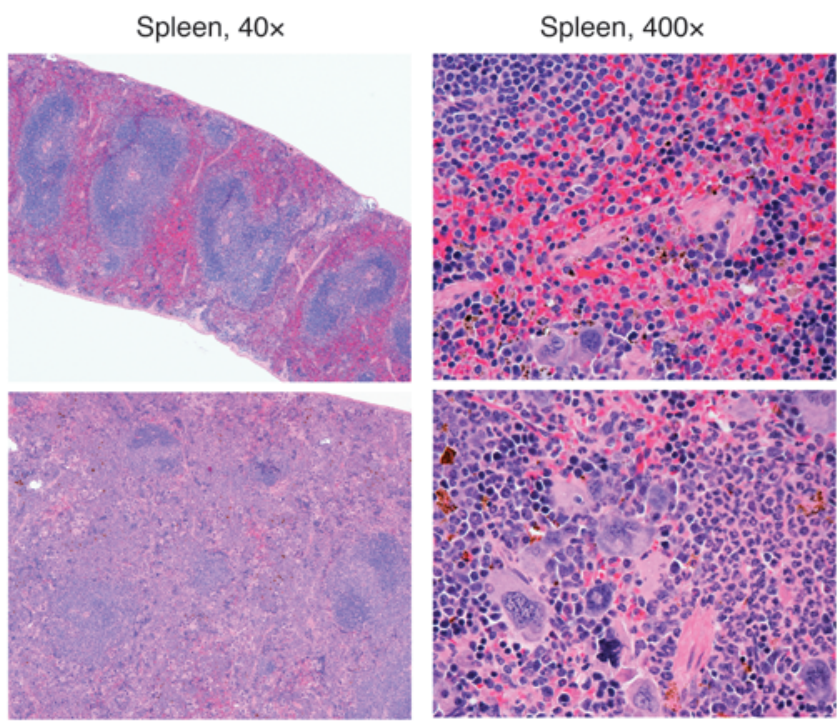

\section{Figure 8}

Aged $L n k^{-1-}$ mice develop a CML-like MPD phenotype. (A) Peripheral blood cell counts of young and old $L^{n} k^{-1-}$ mice in comparison with agematched WT mice. Each symbol represents an individual mouse. Average blood counts are indicated by horizontal lines. Young mice are 2 months of age, and old mice are 12-18 months of age. (B) Spleen weight of old WT and $L n k^{-1-}$ mice. Average spleen weights are indicated by horizontal lines. (C) Percentage of Gr-1+Mac-1+ myeloid cells, CD4+/CD8 ${ }^{+} \mathrm{T}$ cells, and B220+ $\mathrm{B}$ cells in the spleen of WT and $L n k^{-1-}$ mice (average \pm SEM). $n=5$. Two-tailed $t$ tests were performed, and $P$ values are indicated on the plots. (D and $\mathbf{E}) \mathrm{H} \& \mathrm{E}$ stainings of the sections from BM located within the femur, spleen, and liver of aged WT and $L n k^{-/-}$mice are shown. Original magnification, $\times 40(\mathbf{D}$, center panel); $\times 200(\mathrm{E}) ; \times 400$ (D, left and right panels). 
part of the signaling machinery that keeps proper control of cell survival, proliferation, and differentiation.

Accumulating evidence points to the importance of the adaptor protein Lnk as a negative regulator of multiple cytokine receptors, such as Mpl, Kit, and EpoR. Lnk constrains normal HSPC expansion in vivo and in vitro. $\mathrm{Lnk}^{-1-}$ progenitor cells give rise to larger colonies in methylcellulose culture and are more sensitive to multiple cytokines in liquid culture (6). However, Lnk deficiency alone is not enough to confer cytokine-independent growth. We show here that loss of Lnk cooperated with other oncogenes to promote myeloid expansion. Of note, Lnk deficiency results in a disproportionate expansion of immature myeloid precursors and sensitizes progenitor cells to oncogene-induced expansion. Yet, this hyperproliferative phenotype fails to trigger blast crises. Furthermore, old Lnk $k^{-/}$mice develop MPDs without any forced oncogene expression, suggesting Lnk restricts myeloid expansion in vivo. The fact that we have not detected any acute myeloid leukemia (AML) in old Lnk- mice is consistent with the notion that Lnk deficiency requires additional oncogenic events to promote blast transformation.

Our data suggest that Lnk deficiency facilitates oncogene-mediated transformation through multiple mechanisms. First, the JAK2V617F/Y813F double mutant that lacks the Lnk binding site conferred superior myeloid expansion and more severe MF in mice, when compared with that of the JAK2V617 mutant alone, suggesting that Lnk directly inhibits JAK2V617F. Second, loss of Lnk facilitates HSPC expansion and self-renewal, thus potentially contributing to cellular transformation by diverse oncogenes. Indeed, Lnk deficiency promotes MPD in the context of BCR/ABL that does not interact with Lnk. This is also consistent with our results showing that WT cells expressing JAK2V617F/Y813F caused a less severe MPD phenotype than Lnk ${ }^{-1-}$;AK2V617F cells. In concert, these findings indicate that loss of Lnk can augment signaling and transformation by exogenous oncogenes, such as JAK2V617F, but also via endogenous signaling pathways in HSPCs that may be dependent on JAK2 or additional targets of Lnk. Thus, our results suggest a broader role of Lnk in hematologic malignancies.

Our studies showing that Lnk deficiency promotes MPD development in mice warrant future investigation of human MPDs. In human MPD patients, Lnk deficiency would be expected to endow JAK2 $2617 \mathrm{~F}^{+}$or BCR/ABL ${ }^{+} \mathrm{HSPCs}$ with a growth advantage over progenitors with WT Lnk. So far, Lnk expression levels have been examined in a few cases of human MPD and CML patients, and the results appeared disparate $(19,32,38)$. Therefore, studies measuring Lnk expression in larger cohorts of human MPD patients are required. Furthermore, it remains to be determined whether Lnk is structurally altered in AML and MPD progenitor cells. As an adaptor protein important for signaling transduction, it might also worth examining Lnk protein modifications, such as phosphorylation, in human leukemia samples.

In summary, we found that Lnk constrains MPD development in mice. Lnk deficiency cooperates with oncogenic JAK2 that binds to Lnk, as well as oncogenes that do not directly bind to Lnk, in causing MPDs. This suggests a broader role of Lnk in hematologic malignancies. Further investigation of the molecular mechanisms by which Lnk attenuates receptor/kinase signaling and identification of novel Lnk targets in both normal and malignant progenitor cells are warranted. Knowledge gained in this report also supports the notion that Lnk is a potential tumor suppressor and that search for Lnk dysregulation should not be restricted to JAK2mediated diseases.

\section{Methods}

Mice. $L n k^{-/-}$mice were provided by Tony Pawson (Samuel Lunenfeld Research Institute, Toronto, Ontario, Canada). WT C57BL/6J mice were purchased from The Jackson Laboratory. All mice were maintained at the Children's Hospital of Philadelphia animal facility under a protocol approved by this institution.

Retroviral constructs. MIG vector was obtained from Keith Humphries at the Terry Fox Laboratory. MIG-TEL/JAK2 and MIG-JAK2V617F constructs were provided by D. Gary Gilliland (Harvard Medical School, Boston, Massachusetts, USA). MSCV-TEL/JAK2-IRES-GFP/Lnk (or LnkRE) was generated by PCR Lnk cDNA and inserted in frame $3^{\prime}$ to the GFP. MIGJAK2V617F/Y813F was generated through site-directed mutagenesis using QuikChange Kit (Stratagene) and confirmed by sequencing.

BM cell isolation and retroviral infection. Eight- to twelve-week-old C57BL6/J and $L n k^{-/-}$mice were injected with 5-FU, 4-5 days prior to BM isolation. BM cells were either spun through a $1.083 \mathrm{~g} / \mathrm{ml}$ ficoll gradient (Sigma-Aldrich) or directly prestimulated with cytokines, RPMI containing 10\% FBS, $2 \mathrm{mM}$ L-glutamine, $100 \mathrm{U} / \mathrm{ml}$ penicillin and streptomycin, $50 \mu \mathrm{M} \beta$-mercaptoethanol, $100 \mathrm{ng} / \mathrm{ml} \mathrm{SCF}, 10 \mathrm{ng} / \mathrm{ml} \mathrm{IL-6}$, and $6 \mathrm{ng} / \mathrm{ml} \mathrm{IL-2} \mathrm{(PeproTech).}$ Subsequently cells were infected with retroviruses by spin inoculation (10, 29). For progenitor cell transplants, Lin- progenitor cells were isolated using StemSep columns (StemCell Technology) or Dynal magnetic beads (Invitrogen), according to manufacturer's protocol. Subsequently Lin- BM cells were prestimulated and spin-infected with the desired viral supernatant in the presence of $10 \mu \mathrm{g} / \mathrm{ml}$ polybrene (Sigma-Aldrich). The infection was repeated on the following day, and infected BM cells were subsequently transplanted into irradiated animals.

$B M T$. Transduced BM cells were injected retro-orbitally into lethally irradiated (a split dose of $10 \mathrm{~Gy},{ }^{137} \mathrm{Cs}$ source) recipient mice. When total BM cells were used, 1 million cells were injected into each recipient mouse. When mononuclear cells (MNNCs) were used, 0.3 million MNNCs from WT donors or 0.1 million from $L n k^{-/-}$donor mice were injected into each recipient mouse. When Lin ${ }^{-} \mathrm{BM}$ progenitor cells were used, $3 \times 10^{4}$ retrovirally transduced progenitor cells were mixed with $2 \times 10^{5}$ freshly isolated competitor cells and injected, unless otherwise indicated. Mice were monitored frequently and moribund animals were euthanized according to the institutional protocol.

Two weeks to four months after transplantation, complete blood count was measured using a Hemavet 950 (Drew Scientific Inc.), and hematocrits were measured on a hematocrit centrifuge (Micro-MB; IEC). Peripheral blood was analyzed for the retrovirally infected $\left(\mathrm{GFP}^{+}\right)$fractions of myeloid, T, and B cell lineages, using flow cytometry. BM and spleen cells from transplanted mice were also analyzed for GFP-positive cells, along with different lineage markers. Specifically, phycoerythrin-conjugated (R-PE-conjugated) anti-Gr-1 and allophycocyanin-conjugated (APC-conjugated) anti-Mac-1 antibodies were used to examine the myeloid lineage. PE-conjugated anti-CD4 and anti-CD8 and APC-conjugated anti-B220 antibodies were used to examine $\mathrm{T}$ and $\mathrm{B}$ cell lineages, respectively. All antibodies were obtained from eBiosciences. Stained cells were resuspended in buffer containing propidium iodide (BD Biosciences) and subjected to flow cytometry analysis, using Becton Dickinson FACSCalibur.

Colony assays. Colony assay was performed in semisolid methylcellulose (StemCell Technologies) according manufacturer's recommendations. Total BM or spleen cells from transplanted mice were plated at appropriate concentrations in M3434 and enumerated 7-10 days later.

$\mathrm{GFP}^{+} \mathrm{Lin}^{-}$progenitor BM cells were purified, using flow cytometric sorting (FACS Aria, BD Biosciences) 2 days after infection, and plated at $2 \times 10^{3}$ cells per dish in cytokine-rich medium (M3434) or $5 \times 10^{3}$ cells per dish in cytokine-free medium (M3234). Eight to ten days later, colony numbers were scored under light microscope. Serial replating assays were performed 
as previously described (39-43). In brief, primary colonies were recovered from the methylcellulose medium. Cells were washed 3 times with Iscove's modified Dulbecco's medium (Invitrogen) with $2 \%$ FBS, and viable cells were enumerated with trypan blue. Under identical conditions, 30,000 cells $/ \mathrm{ml}$ were replated in each secondary plate. Colonies were scored after another 8-10 days.

Cell growth assay. Purified $\mathrm{GFP}^{+} \mathrm{Lin}^{-} \mathrm{BM}$ progenitor cells were plated at equal concentrations in a 96-well plate in RPMI medium, supplemented with $10 \%$ FBS only (cytokine free) or with IL-3 (10\% WEHI supernatant). Cells were counted and renewed every 2-4 days in trypan blue.

Histology and cytology. Femurs, tibias, spleens, and livers from transplanted mice were fixed in 10\% formalin (Sigma-Aldrich) and processed for paraffin blocks and sections. H\&E stainings were used for histology. Reticulin stainings were used to detect reticulin fibers in the spleen and BM tissues. Blood smears were fixed in cold methanol and stained with Wright-Giemsa (EMD Chemicals), according to manufacturer's recommendation. Cytology and histology images were taken using a Leica DM4000B microscope, with plan FL2 $\times 10, \times 20$, and $\times 40$ objective lenses, and a Spot RT/SE Slider digital camera from SPOT Imaging Solutions.

Protein lysates and Western blot analysis. BM and spleen cells from transplanted mice were isolated and starved in RPMI with $0.5 \%$ BSA for 1-2 hours. The cells were either left unstimulated or stimulated with $10 \mathrm{ng} / \mathrm{ml}$ of Tpo or $1 \mathrm{ng} / \mathrm{ml}$ of IL-3 for 10 minutes. Subsequently cells were lysed in $10 \mathrm{mM}$ Tris- $\mathrm{Cl}$ ( $\mathrm{pH} 7.4), 150 \mathrm{mM} \mathrm{NaCl}$, and $1 \% \mathrm{NP}-40$, containing $2 \mathrm{mM}$ each of $\mathrm{NaF}$ and $\mathrm{Na}_{3} \mathrm{~V}_{2} \mathrm{O}_{4}$ plus protease inhibitors (Roche). The samples were fractionated through Bis-Tris NuPAGE gels (Novex, Invitrogen) and transferred onto Nitrocellulose membranes (Schleicher \& Schuell Bioscience). The blots were then probed with the indicated antibodies: anti-Stat5 (C-17) rabbit polyclonal antibodies (1:500, Santa Cruz Technology Inc.) and antip-Stat5 (Tyr694), p-MAPK (Thr202/Tyr204), p42/44 MAPK, pAkt (Thr473), and Akt antibodies (1:1,000, all from Cell Signaling Technology).

Statistics. Two-tailed Student's $t$ tests were used. A P value of less than 0.05 was considered significant.

\section{Acknowledgments}

W. Tong was supported by the Howard Temin Career Development award from NCI (K01CA115679). This work was supported by NIH grants R01HL095675 (to W. Tong) and R01DK054937 (to G.A. Blobel). A. Bersenev was supported by an NIH training grant (T32HL007971). We are grateful to Tony Pawson for providing us $L n k^{-/-}$mice, and Nancy Speck and Roger Greenberg for critical review of the manuscript.

Received for publication December 14, 2009, and accepted in revised form March 31, 2010.

Address correspondence to: Wei Tong, Abramson Bldg., Suite 316A, Children's Hospital of Philadelphia, 3615 Civic Center Boulevard, Philadelphia, Pennsylvania 19104, USA. Phone: 267.426.0930; Fax: 267.426.5476; E-mail: tongw@email.chop.edu.
1. Watowich SS, Wu H, Socolovsky M, Klingmuller U, Constantinescu SN, Lodish HF. Cytokine receptor signal transduction and the control of hematopoietic cell development. Annu Rev Cell Dev Biol. 1996;12:91-128.

2. Drachman JG, Kaushansky K. Structure and function of the cytokine receptor superfamily. Curr Opin Hematol. 1995;2(1):22-28.

3. Schlessinger J, Lemmon MA. SH2 and PTB domains in tyrosine kinase signaling. Sci STKE. 2003; 2003(191):RE12.

4. Rudd CE. Lnk adaptor: novel negative regulator of B cell lymphopoiesis. Sci STKE. 2001;2001(85):pe1.

5 . Takaki S, et al. Control of B cell production by the adaptor protein lnk. Definition Of a conserved family of signal-modulating proteins. Immunity. 2000; 13(5):599-609.

6. Velazquez L, et al. Cytokine signaling and hematopoietic homeostasis are disrupted in Lnk-deficient mice. J Exp Med. 2002;195(12):1599-1611.

7. Ema H, et al. Quantification of self-renewal capacity in single hematopoietic stem cells from normal and Lnk-deficient mice. Dev Cell. 2005;8(6):907-914.

8. Bersenev A, Wu C, Balcerek J, Tong W. Lnk controls mouse hematopoietic stem cell self-renewal and quiescence through direct interactions with JAK2. JClin Invest. 2008;118(8):2832-2844.

9. Buza-Vidas N, et al. Cytokines regulate postnatal hematopoietic stem cell expansion: opposing roles of thrombopoietin and LNK. Genes Dev. 2006; 20(15):2018-2023.

10. Tong W, Lodish HF. Lnk inhibits Tpo-mpl signaling and Tpo-mediated megakaryocytopoiesis. J Exp Med. 2004;200(5):569-580.

11. Seita J, et al. Lnk negatively regulates self-renewal of hematopoietic stem cells by modifying thrombopoietin-mediated signal transduction. Proc Natl Acad Sci U S A. 2007;104(7):2349-2354.

12. Dameshek W. Some speculations on the myeloproliferative syndromes. Blood. 1951;6(4):372-375.

13. Tefferi A, Gilliland DG. Oncogenes in myeloproliferative disorders. Cell Cycle. 2007;6(5):550-566.

14. Lacronique $V$, et al. A TEL-JAK2 fusion protein with constitutive kinase activity in human leukemia. Sci- ence. 1997;278(5341):1309-1312.

15. Peeters $P$, et al. Fusion of TEL, the ETS-variant gene 6 (ETV6), to the receptor-associated kinase JAK2 as a result of $\mathrm{t}(9 ; 12)$ in a lymphoid and $\mathrm{t}(9 ; 15 ; 12)$ in a myeloid leukemia. Blood. 1997;90(7):2535-2540.

16. James $\mathrm{C}$, et al. A unique clonal JAK2 mutation leading to constitutive signalling causes polycythaemia vera. Nature. 2005;434(7037):1144-1148.

17. Kralovics R, et al. A gain-of-function mutation of JAK2 in myeloproliferative disorders. N Engl J Med. 2005;352(17):1779-1790.

18. Levine RL, et al. Activating mutation in the tyrosine kinase JAK2 in polycythemia vera, essential thrombocythemia, and myeloid metaplasia with myelofibrosis. Cancer Cell. 2005;7(4):387-397.

19. Gery S, Cao Q, Gueller S, Xing H, Tefferi A, Koeffler HP. Lnk inhibits myeloproliferative disorderassociated JAK2 mutant, JAK2V617F. J Lenkoc Biol. 2009;85(6):957-965.

20. Gery S, Gueller S, Chumakova K, Kawamata N, Liu L, Koeffler HP. Adaptor protein Lnk negatively regulates the mutant MPL, MPLW515L associated with myeloproliferative disorders. Blood. 2007; 110(9):3360-3364.

21. Argiropoulos B, Humphries RK. Hox genes in hematopoiesis and leukemogenesis. Oncogene. 2007; 26(47):6766-6776.

22. Hock H, Orkin SH. Zinc-finger transcription factor Gfi-1: versatile regulator of lymphocytes, neutrophils and hematopoietic stem cells. Curr Opin Hematol. 2006;13(1):1-6.

23. Krivtsov AV, Armstrong SA. MLL translocations, histone modifications and leukaemia stem-cell development. Nat Rev Cancer. 2007;7(11):823-833.

24. Pardal R, Molofsky AV, He S, Morrison SJ. Stem cell self-renewal and cancer cell proliferation are regulated by common networks that balance the activation of proto-oncogenes and tumor suppressors. Cold Spring Harb Symp Quant Biol. 2005;70:177-185.

25. Rossi DJ, Weissman IL. Pten, tumorigenesis, and stem cell self-renewal. Cell. 2006;125(2):229-231.

26. Schwaller J, et al. Transformation of hematopoietic cell lines to growth-factor independence and induction of a fatal myelo- and lymphoproliferative dis- ease in mice by retrovirally transduced TEL/JAK2 fusion genes. Embo J. 1998;17(18):5321-5333.

27. Ueda Y, Kondo M, Kelsoe G. Inflammation and the reciprocal production of granulocytes and lymphocytes in bone marrow. J Exp Med. 2005; 201(11):1771-1780.

28. Terskikh AV, Miyamoto T, Chang C, Diatchenko L, Weissman IL. Gene expression analysis of purified hematopoietic stem cells and committed progenitors. Blood. 2003;102(1):94-101.

29. Tong W, Zhang J, Lodish HF. Lnk inhibits erythropoiesis and Epo-dependent JAK2 activation and downstream signaling pathways. Blood. 2005; 105(12):4604-4612.

30. Wernig G, Mercher T, Okabe R, Levine RL, Lee BH, Gilliland DG. Expression of Jak2V617F causes a polycythemia vera-like disease with associated myelofibrosis in a murine bone marrow transplant model. Blood. 2006;107(11):4274-4281.

31. Lacout C, Pisani DF, Tulliez M, Gachelin FM, Vainchenker W, Villeval JL. JAK2V617F expression in murine hematopoietic cells leads to MPD mimicking human PV with secondary myelofibrosis. Blood. 2006;108(5):1652-1660.

32. Gery S, Gueller S, Nowak V, Sohn J, Hofmann WK, Koeffler HP. Expression of the adaptor protein Lnk in leukemia cells. Exp Hematol. 2009;37(5):585-592.e2.

33. Klingmuller U, Lorenz U, Cantley LC, Neel BG, Lodish HF. Specific recruitment of SH-PTP1 to the erythropoietin receptor causes inactivation of JAK2 and termination of proliferative signals. Cell. 1995;80(5):729-738.

34. Rathinam C, Thien CB, Langdon WY, Gu H, Flavell RA. The E3 ubiquitin ligase c-Cbl restricts development and functions of hematopoietic stem cells. Genes Dev. 2008;22(8):992-997.

35. Sanada M, et al. Gain-of-function of mutated C-CBL tumour suppressor in myeloid neoplasms. Nature. 2009;460(7257):904-908.

36. Inagaki-Ohara K, Hanada T, Yoshimura A. Negative regulation of cytokine signaling and inflammatory diseases. Curr Opin Pharmacol. 2003;3(4):435-442.

37. Krebs DL, Hilton DJ. SOCS: physiological suppressors of cytokine signaling. J Cell Sci. 2000; 
113(pt 16):2813-2819.

38. Lemoli RM, et al. Molecular and functional analysis of the stem cell compartment of chronic myelogenous leukemia reveals the presence of a CD34-cell population with intrinsic resistance to imatinib. Blood. 2009;114(25):5191-5200.

39. Will B, et al. Effect of the nonpeptide thrombopoietin receptor agonist Eltrombopag on bone marrow cells from patients with acute myeloid leuke- mia and myelodysplastic syndrome. Blood. 2009; 114(18):3899-3908.

40. Steidl U, et al. Essential role of Jun family transcription factors in PU.1 knockdown-induced leukemic stem cells. Nat Genet. 2006;38(11):1269-1277.

41. Huntly BJ, et al. MOZ-TIF2, but not BCR-ABL, confers properties of leukemic stem cells to committed murine hematopoietic progenitors. Cancer Cell. 2004;6(6):587-596.
42. Higuchi M, O’Brien D, Kumaravelu P, Lenny N, Yeoh EJ, Downing JR. Expression of a conditional AML1ETO oncogene bypasses embryonic lethality and establishes a murine model of human $\mathrm{t}(8 ; 21)$ acute myeloid leukemia. Cancer Cell. 2002;1(1):63-74.

43. Lavau C, Szilvassy SJ, Slany R, Cleary ML. Immortalization and leukemic transformation of a myelomonocytic precursor by retrovirally transduced HRX-ENL. EMBO J. 1997;16(14):4226-4237. 